A LETTERS JOURNAL EXPLORING THE FRONTIERS OF PHYSICS

\title{
OFFPRINT
}

\section{Landau-Zener and Rabi oscillations in the spin-dependent conductance}

\author{
L. J. Fernández-Alcázar and H. M. Pastawski \\ EPL, 105 (2014) 17005
}

Please visit the new website

www.epl journal.org 


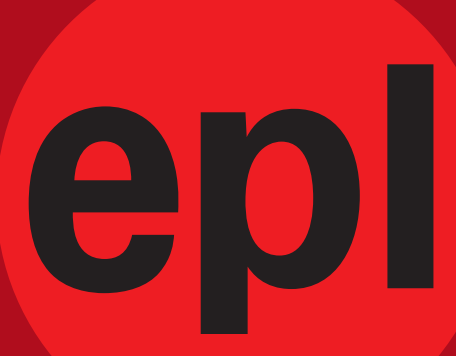

A LetTers JoUrnal EXPLoring the Frontiers of Physics

\section{AN INVITATION TO SUBMIT YOUR WORK}

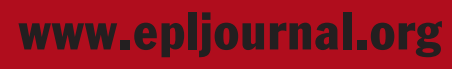

The Editorial Board invites you to submit your letters to EPL

EPL is a leading international journal publishing original, high-quality Letters in all areas of physics, ranging from condensed matter topics and interdisciplinary research

to astrophysics, geophysics, plasma and fusion sciences, including those with application potential.

The high profile of the journal combined with the excellent scientific quality of the articles continue to ensure EPL is an essential resource for its worldwide audience. EPL offers authors global visibility and a great opportunity to share their work with others across the whole of the physics community.

\section{Run by active scientists, for scientists}

EPL is reviewed by scientists for scientists, to serve and support the international scientific community. The Editorial Board is a team of active research scientists with an expert understanding of the needs of both authors and researchers.
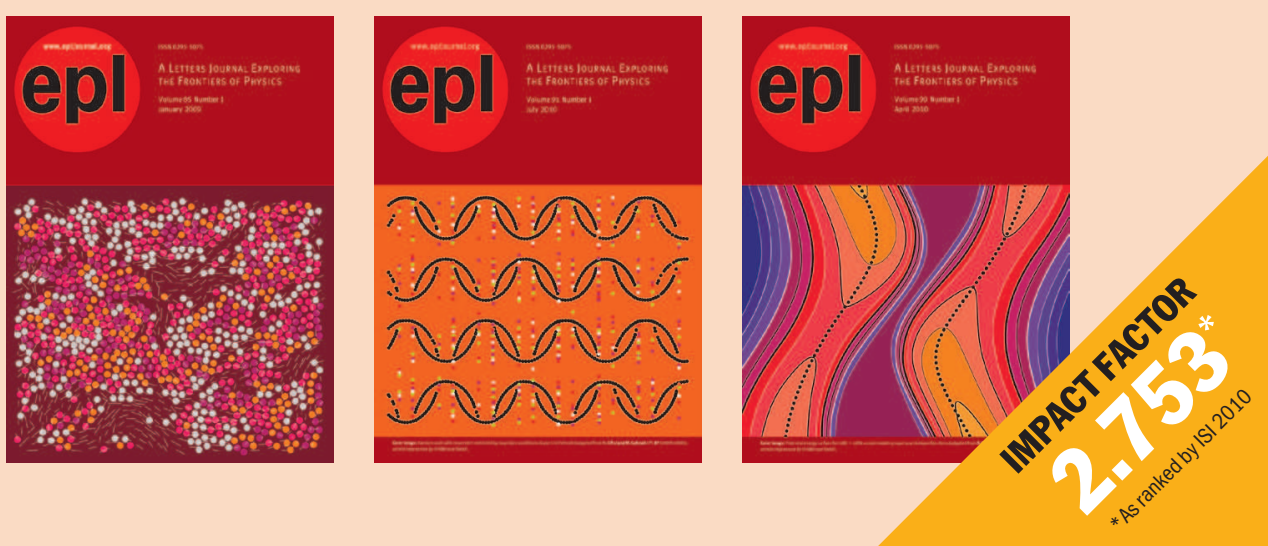

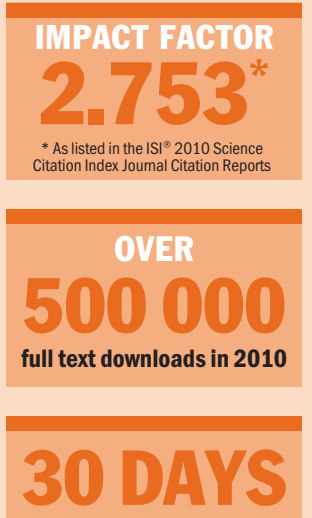

average receipt to online publication in 2010

\section{(1) $0 \longdiv { 6 }$ \\ citations in $\mathbf{2 0 1 0}$ \\ $37 \%$ increase from 2007}

"We've had a very positive experience with EPL, and not only on this occasion. The fact that one can identify an appropriate editor, and the editor is an active scientist in the field, makes a huge difference."

Dr. Ivar Martinv

Los Alamos National Laboratory, USA

\section{Six good reasons to publish with EPL}

We want to work with you to help gain recognition for your high-quality work through worldwide visibility and high citations.

Quality - The 40+ Co-Editors, who are experts in their fields, oversee the entire peer-review process, from selection of the referees to making all final acceptance decisions

Impact Factor - The 2010 Impact Factor is 2.753; your work will be in the right place to be cited by your peers

Speed of processing - We aim to provide you with a quick and efficient service; the median time from acceptance to online publication is 30 days

High visibility - All articles are free to read for 30 days from online publication date

International reach - Over 2,000 institutions have access to EPL, enabling your work to be read by your peers in 100 countries

Open Access - Articles are offered open access for a one-off author payment

Details on preparing, submitting and tracking the progress of your manuscript from submission to acceptance are available on the EPL submission website www.epletters.net.

If you would like further information about our author service or EPL in general, please visit www.epljournal.org or e-mail us at info@epljournal.org.

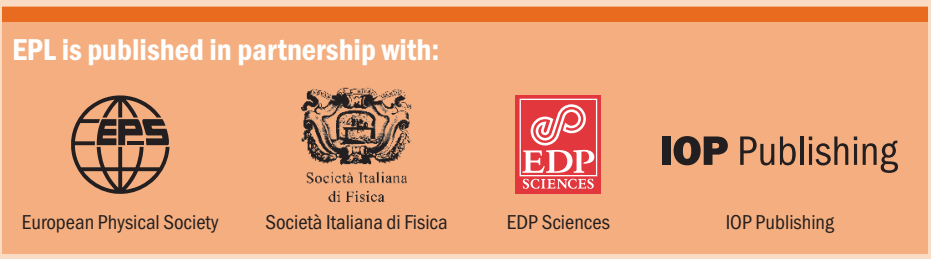

\section{www.epljournal.org}




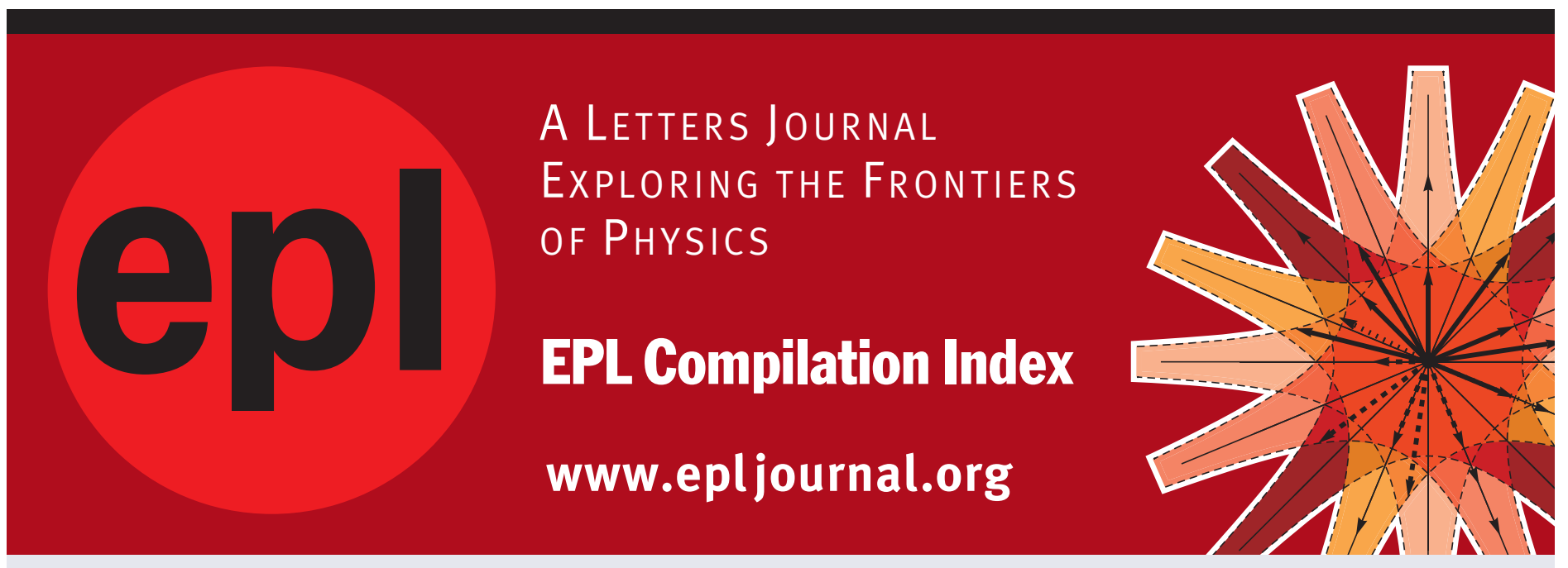

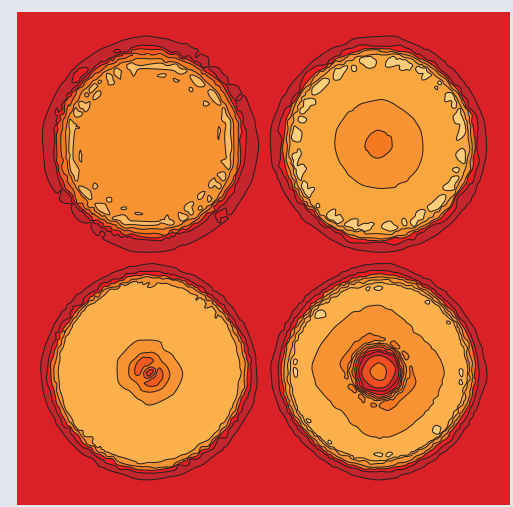

Biaxial strain on lens-shaped quantum rings of different inner radii, adapted from Zhang et al 2008 EPL 8367004.

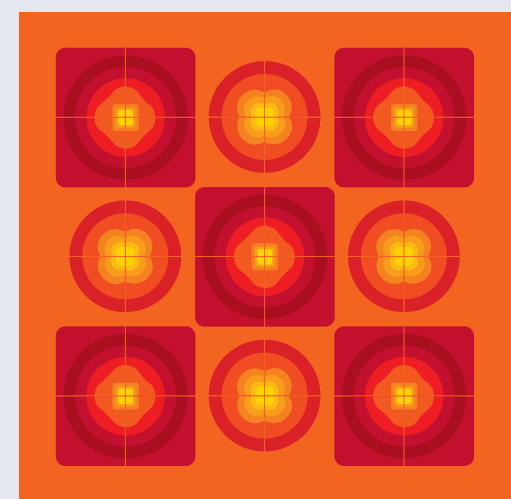

Artistic impression of electrostatic particle-particle interactions in dielectrophoresis, adapted from N Aubry and P Singh 2006 EPL 74623.

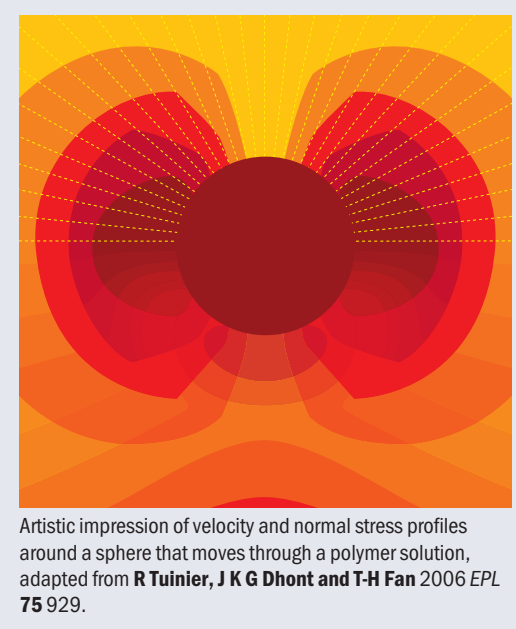

Visit the EPL website to read the latest articles published in cutting-edge fields of research from across the whole of physics.

Each compilation is led by its own Co-Editor, who is a leading scientist in that field, and who is responsible for overseeing the review process, selecting referees and making publication decisions for every manuscript.

- Graphene

- Liquid Crystals

- High Transition Temperature Superconductors

- Quantum Information Processing \& Communication

- Biological \& Soft Matter Physics

- Atomic, Molecular \& Optical Physics

- Bose-Einstein Condensates \& Ultracold Gases

- Metamaterials, Nanostructures \& Magnetic Materials

- Mathematical Methods

- Physics of Gases, Plasmas \& Electric Fields

- High Energy Nuclear Physics

If you are working on research in any of these areas, the Co-Editors would be delighted to receive your submission. Articles should be submitted via the automated manuscript system at www.epletters.net

If you would like further information about our author senvice or EPL in general, please visit www.epljournal.org or e-mail us at info@epljournal.org

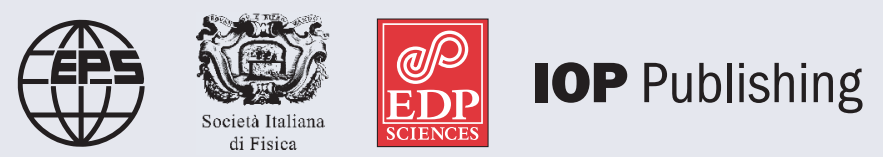

Image: Ornamental multiplication of space-time figures of temperature transformation rules (adapted from T. S. Bíró and P. Ván 2010 EPL 89 30001; artistic impression by Frédérique Swist). 


\title{
Landau-Zener and Rabi oscillations in the spin-dependent conductance
}

\author{
L. J. Fernández-Alcázar and H. M. Pastawski
}

Instituto de Física Enrique Gaviola, CONICET-UNC - Córdoba, Argentina and Facultad de Matemática, Astronomía y Física, Universidad Nacional de Córdoba Ciudad Universitaria, 5000 Córdoba, Argentina

received 18 November 2013; accepted in final form 3 January 2014

published online 3 February 2014

PACS 72.25.-b - Spin polarized transport

PACS 72.25.Ba - Spin polarized transport in metals

PACS 85.75.-d-Magnetoelectronics; spintronics: devices exploiting spin polarized transport or integrated magnetic fields

\begin{abstract}
We describe the spin-dependent quantum conductance in a wire where a magnetic field is spatially modulated. The change in direction and intensity of the magnetic field acts as a perturbation that mixes spin projections. This is exemplified by a ferromagnetic nanowire. There the local field varies smoothly its direction generating a domain wall (DW) as described by the well-known Cabrera-Falicov model. Here, we generalize this model to include also a strength modulation. We identify two striking diabatic regimes that appear when such magnetic inhogeneity occurs. 1) If the field strength at the DW is weak enough, the local Zeeman energies result in an avoided crossing. Thus, the spin-flip probability follows the Landau-Zener formula. 2) For strong fields, the spin-dependent conductance shows oscillations as a function of the DW width. We interpret them in terms of Rabi oscillations. Time and length scales obtained from this simplified view show an excellent agreement with the exact dynamical solution of the spin-dependent transport. These results remain valid for other situations involving modulated magnetic structures and thus they open new prospects for the use of quantum interferences in spin-based devices.
\end{abstract}

This paper is dedicated to the memory of the lifelong collaborator Patricia Rebeca Levstein.

Copyright (c) EPLA, 2014

Introduction. - The control and design of spindependent electronic transport in magnetically modulated devices represents a promising technological challenge [1]. Spintronic devices switch the spin state or filter electrons by spin. The most direct way to tune the transport, apart from spin-orbit effects [2,3], is to use designed magnetic inhomogeneities which couple directly to the spin. The most prominent devices are those based on the giant magnetoresistance [4]. Other recent developments involve spin valves based on organic molecules $[5,6]$, and quasi-onedimensional spin transistors $[7,8]$. Since quantum effects become relevant, transport is based on Landauer's motto: "conductance is transmittance" [9].

Previous studies of spin-dependent quantum transport suggest the presence of interesting physical phenomena. For example, conductance through ferromagnetic nanowires with a domain wall (DW) shows some FabryPérot like interferences, which were not fully understood $[10,11]$. Also, transport on magnetically modulated semiconducting spin valves [8] showed magnetic commensurabilities as well as regimes compatible with a Landau-Zener problem. Thus, quantum transport through magnetic inhomogeneities becomes a promising tool in spintronics, where the different characteristic times and lengths should be identified. These scales should be compared with the electron's Fermi wavelength. The tunneling adiabaticity is given by the electron's speed. In this context, a dynamical description of the transport process would improve the comprehension of these phenomena.

In this letter we consider a variant of the Cabrera and Falicov [12] model for spin-dependent electronic transport through a soft magnetic DW. It is representative of a wide class of magnetic inhomogeneities. In the original model the field just rotates along the DW. We extend it allowing a modulation in the field strength. This simple variation will have nontrivial consequences on transport. We show that in a weak perturbation regime, the spindependent conductance through the DW can be described 
by the Landau-Zener (LZ) formula. In contrast, for a strong perturbation regime, we find well-defined interferences as a function of the DW width, which are interpreted as Rabi oscillations. This interpretation is confirmed by an analysis of the wave packet dynamics. The physics and computational strategies described here could help in the design of better spintronic devices.

Hamiltonian of the conduction electrons. - We consider a single-spin channel [10]:

$$
\hat{H}=-\frac{\hbar^{2}}{2 m} \frac{\mathrm{d}^{2}}{\mathrm{~d} x^{2}}-\vec{\mu} \cdot \vec{B}(x) .
$$

The first term is the kinetic energy along $x$ of electrons with effective mass $m$. The second term is the Zeeman interaction between the spin magnetic moment $\vec{\mu}$ and $\vec{B}(x)$, the effective magnetic field at $x$. Here, $\vec{\mu}=-\mu_{B} \vec{\sigma}$, where $\mu_{B}$ is the Bohr magneton and $\vec{\sigma}=\left(\sigma_{x}, \sigma_{y}, \sigma_{z}\right)$ is the vector of the Pauli matrices. In particular, the dependence on $x$ of the magnitude and direction of $\vec{B}(x)$ may cause the spin-dependent scattering. These inhomogeneities may be natural, as in ferromagnetic DWs [13], or artificially generated, as in magnetic semiconducting waveguides [7]. We will express our results in the concrete language of ferromagnetic nanowires.

Electronic structure in modulated magnetic fields. - The Cabrera and Falicov soft-DW model [12] considers a quantum spin channel laid along $x$, and a magnetic field whose orientation rotates as it progress along the DW. We generalize this description by including a modulation in the field intensity. The vector $\vec{f}(x)=\left(f_{x}(x), f_{y}(x), f_{z}(x)\right)=-\vec{B}(x) / B_{\infty}$, describes the DW shape, and satisfies $|\vec{f}(x)| \rightarrow 1$ as $x \longrightarrow \pm \infty$. The asymmetry in the modulation strength is described by the parameter $\alpha=B(0) / B_{\infty}$. Using the axis $z$ as quantization direction for the spin,

$$
\vec{f}(x)=\left(\alpha / \cosh \left(\frac{x}{W}\right), \quad 0, \quad \tanh \left(\frac{x}{W}\right)\right) .
$$

Here, $W$ is the half-width of the DW. Obviously, $\alpha=1$ corresponds to a field of constant strength that rotates across the DW. The Zeeman term is $\vec{\mu} \cdot \vec{B}(x)=\Delta_{0} \vec{\sigma} \cdot \vec{f}(x)$. Here, $\Delta_{0}=\mu_{B} B_{\infty}$. The wave function for a conduction electron has components along both spin directions, $|\uparrow\rangle$ and $|\downarrow\rangle$, referred to the quantization axis parallel to the field at the left domain (laboratory frame), as

$$
|\psi(x)\rangle=\varphi_{\uparrow}(x)|x, \uparrow\rangle+\varphi_{\downarrow}(x)|x, \downarrow\rangle .
$$

Therefore, the equations governing the electron tunneling and the spin flip are

$$
\left\{\begin{array}{l}
-\frac{\hbar^{2}}{2 m} \frac{\mathrm{d}^{2}}{\mathrm{~d} x^{2}} \varphi_{\uparrow}(x)+E_{\uparrow}(x) \varphi_{\uparrow}(x)+V_{\uparrow \downarrow}(x) \varphi_{\downarrow}(x)=\varepsilon \varphi_{\uparrow}(x), \\
-\frac{\hbar^{2}}{2 m} \frac{\mathrm{d}^{2}}{\mathrm{~d} x^{2}} \varphi_{\downarrow}(x)-E_{\downarrow}(x) \varphi_{\downarrow}(x)+V_{\uparrow \downarrow}(x) \varphi_{\uparrow}(x)=\varepsilon \varphi_{\downarrow}(x),
\end{array}\right.
$$

where $\varepsilon$ is the energy associated with the dynamics along $x$, identifying,

$$
\mu_{B} B_{z}(x)=E_{\downarrow}(x)=\Delta_{0} \tanh \left(\frac{x}{W}\right),
$$

and

$$
\mu_{B} B_{x}(x)=V_{\uparrow \downarrow}(x)=\alpha \Delta_{0} / \cosh \left(\frac{x}{W}\right)
$$

where $E_{\downarrow}(x)=-E_{\uparrow}(x)$. The local states become mixed by $V_{\uparrow \downarrow}(x)$ while the electron moves through the DW.

Evaluation of the conductance. - To evaluate the quantum conductance we will use the Landauer-Büttiker equation [14]. There, different conductances are given by the transmittances between states of definite momentum and spin projection at the contacts [9].

In a tight-binding representation [15], the spatial coordinate takes discrete values $x_{n}$ in a grid of unit $a, x_{n} \rightarrow n a$. Every site in the grid has an associated normalized local wave function $|n\rangle$, which will be called $n$-th orbital as in a LCAO scheme. Each orbital has an energy given by the local potential $E_{s}\left(x_{n}\right)=E_{n, s}$, where $s$ is either $\uparrow$ or $\downarrow$, and the transverse field yields $V_{\uparrow \downarrow}\left(x_{n}\right)=V_{n, \uparrow \downarrow}$. Any electronic wave function with well-defined spin $s$ is now written in terms of a discrete sum:

$$
\left|\varphi_{s}\right\rangle \rightarrow \sum_{n} u_{n, s}|n, s\rangle
$$

where, according to eq. (4), the spin-orbital amplitudes $u_{n, s}$ must satisfy

$$
\left\{\begin{array}{r}
-V\left[u_{n+1, \uparrow}-2 u_{n, \uparrow}+u_{n-1, \uparrow}\right]+E_{n, \uparrow} u_{n, \uparrow} \\
+V_{n, \uparrow \downarrow} u_{n+1, \downarrow}=\varepsilon u_{n, \uparrow}, \\
-V\left[u_{n+1, \downarrow}-2 u_{n, \downarrow}+u_{n-1, \downarrow}\right]+E_{n, \downarrow} u_{n, \downarrow} \\
+V_{n, \uparrow \downarrow} u_{n+1, \uparrow}=\varepsilon u_{n, \downarrow} .
\end{array}\right.
$$

The unit of energy is given by the hopping strength $V=\hbar^{2} /\left(2 m a^{2}\right)$. We consider energies at the band center $(\varepsilon \simeq 2 V)$. Each spin orientation is represented by a chain with $N \gg 1$ orbitals that comprises the whole DW. $L$ and $R$ are sites indices symmetrically arranged at the left and right sides of the DW and satisfying $(R-L) a=N a \gg W$. Then $E_{n, s} \equiv E_{L, s}= \pm \Delta_{0}$ for $n \leq L$ and $E_{n, s} \equiv E_{R, s}=\mp \Delta_{0}$ for $n \geq R$. Since far away from the DW, $V_{\uparrow \downarrow} \equiv 0$, the asymptotic eigenvalues, with wave vector $k$, are $\varepsilon_{k, s}= \pm \Delta_{0}+2 V-2 V \cos (k a)$. In the region of the DW both spin orientations become coupled by the perpendicular component of the magnetic field, represented by the hopping element $V_{n, \uparrow \downarrow}$.

The magnetic domains, which play the role of contacts, are described through a renormalization procedure [16]. In an open system it leads to a non-Hermitian effective Hamiltonian [17]:

$$
\hat{H}_{T}=\hat{H}+\hat{\Sigma},
$$



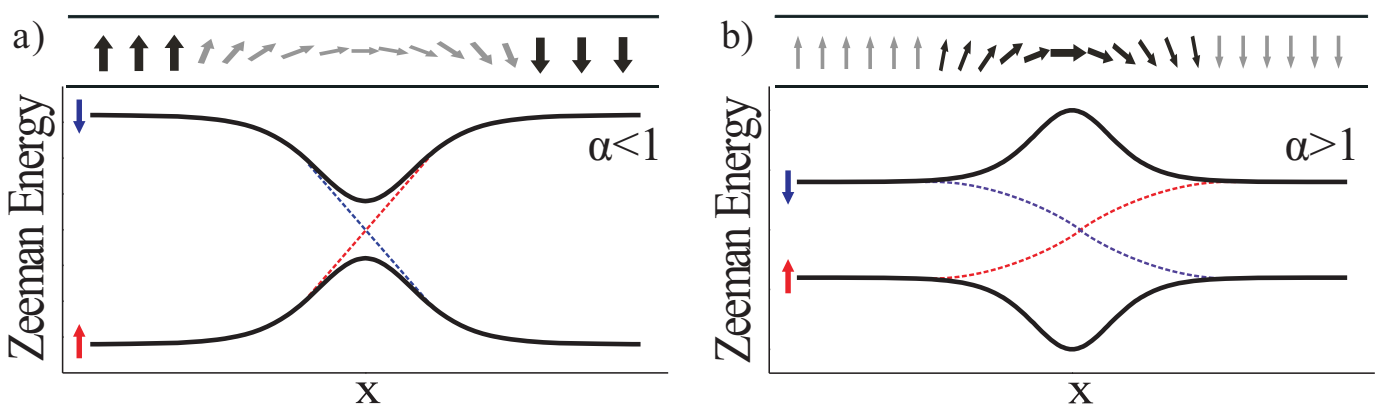

Fig. 1: (Color online) Solid black lines are the local eigenenergies for electrons with $\uparrow$ and $\downarrow$ spin in the presence of a locally rotated field which is schematized on top. (a) Weak $(\alpha<1)$ or (b) strong $(\alpha>1)$ field strength at the DW center. Dashed lines are the unperturbed Zeeman energies.

where

$$
\begin{aligned}
\hat{\Sigma}(\varepsilon) & =\Sigma_{L \uparrow}(\varepsilon)|L \uparrow\rangle\left\langle L \uparrow\left|+\Sigma_{L \downarrow}(\varepsilon)\right| L \downarrow\right\rangle\langle L \downarrow| \\
& +\Sigma_{R \uparrow}(\varepsilon)|R \uparrow\rangle\left\langle R \uparrow\left|+\Sigma_{R \downarrow}(\varepsilon)\right| R \downarrow\right\rangle\langle R \downarrow| .
\end{aligned}
$$

$\Sigma_{j}$ are the self-energies that satisfy the Dyson equation in the magnetic domains:

$$
\begin{aligned}
\Sigma_{j}(\varepsilon) & =\frac{V^{2}}{\varepsilon-E_{j}-\Sigma_{j}(\varepsilon)}=\operatorname{Re} \Sigma_{j}(\varepsilon)-\mathrm{i} \Gamma_{j}(\varepsilon) \\
& \simeq-\mathrm{i} \Gamma_{j}, \quad \text { at the band center. }
\end{aligned}
$$

The double spin-orbital subscript $j=L \uparrow, L \downarrow, R \uparrow$ or $R \downarrow$ indicates the left $(L)$ or right $(R)$ channels inside the magnetic domains with the corresponding spin orientation. $2 a \Gamma_{j} / \hbar$ is the group velocity at the spin channels $j$ connected to each spin orbital at the sides, i.e. $L \uparrow, L \downarrow, R \uparrow$ and $R \downarrow$ in a four-terminal circuit.

We obtain the retarded and advanced Green functions from $\hat{H}_{T}$ as $\hat{G}^{R}(\varepsilon)=\lim _{\eta \rightarrow 0^{+}}\left(\varepsilon+\mathrm{i} \eta-\hat{H}_{T}\right)^{-1}$ and $\hat{G}^{A}=$ $\hat{G}^{R \dagger}$. The transmittance is [15]

$$
T_{i j}(\varepsilon)=2 \Gamma_{i}(\varepsilon)\left|G_{i j}^{R}(\varepsilon)\right|^{2} 2 \Gamma_{j}(\varepsilon) .
$$

Here, $\Gamma_{j}=\operatorname{Im}\left(\Sigma_{j}\right)$ and $i, j=L \uparrow, L \downarrow, R \uparrow, R \downarrow$, being $j$ and $i$ the electronic input and output spin-orbital channels, respectively. When the evaluated channels correspond to opposite spin projections in opposite sides of the DW we call them spin-flip transmittances, e.g., $T_{\downarrow \uparrow}$.

Transport in the regime of $\alpha<1$ as a LandauZener problem. - In eq. (4) $V_{\uparrow \downarrow}$ is responsible for the mixture of the spin orientations. While $V_{\uparrow \downarrow}(x)$ vanishes within the domains, it is roughly constant at the DW center. Besides, $E_{\uparrow}$ and $E_{\downarrow}$ account for the Zeeman energy in the laboratory frame and they have the meaning of effective potentials for those electrons oriented along the field. While, inside the domains, $E_{\uparrow}-E_{\downarrow}=2 \Delta_{0}$ quantifies the Zeeman splitting, at the DW center both energies intersect $E_{\uparrow}=E_{\downarrow}=0$.

Consider an electron wave packet with a given spin projection that moves with definite momentum towards the DW. Its mean position results proportional to the elapsed

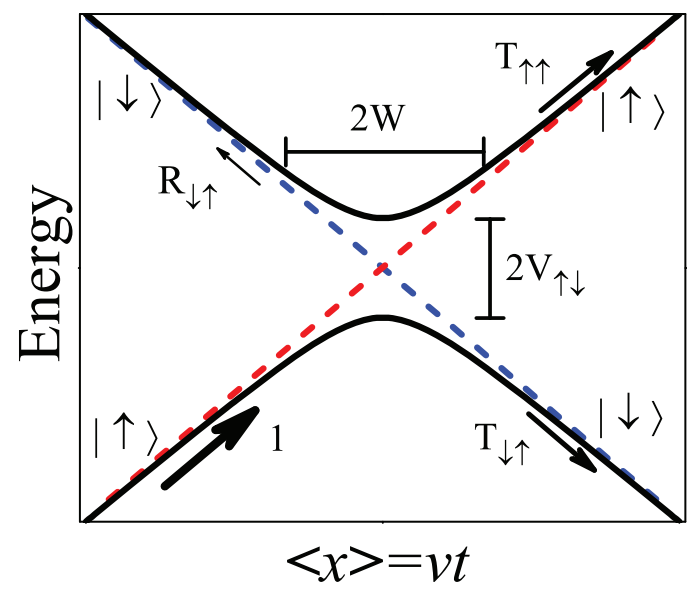

Fig. 2: (Color online) Scheme of the eigenenergies of a two-level system, $|\uparrow\rangle$ and $|\downarrow\rangle$, as functions of the dynamical parameter, $x=v t$, that controls the Landau-Zener transition. The energy levels show an avoided crossing due to the presence of a perturbation that couples the states. The unperturbed energies are shown with dashed lines. Energy and coordinate have arbitrary dimensions.

time $\langle x(t)\rangle \equiv x \simeq v_{F} t$. The spin-dependent mean potential energy will change as the wave packet moves through the DW and starts to be mixed with that of the opposite spin by the $V_{\uparrow \downarrow}$ term. These potential energies are assimilable to the time-dependent local energies of a two level system in a LZ problem [18]. This last describes the transition between two states when their unperturbed energies are swept across a degeneracy point while a constant perturbation produces an avoided crossing. In fig. 1 we show the local Zeeman levels for the DW region. The comparison between the levels of fig. 1(a) and the energy diagram of the two-level system, shown in fig. 2, evidences the analogy between the $\alpha<1$ regime and the LZ problem. Thus, the LZ equation predicts that the probability to exit in the state $|\downarrow\rangle$ to the right, provided that it entered from the left in the state $|\uparrow\rangle$, is

$$
P_{\downarrow \uparrow}=1-\exp \left(-\frac{2 \pi}{\hbar} \frac{\left|V_{\uparrow \downarrow}\right|^{2}}{(\mathrm{~d} E(0) / \mathrm{d} t)}\right) .
$$




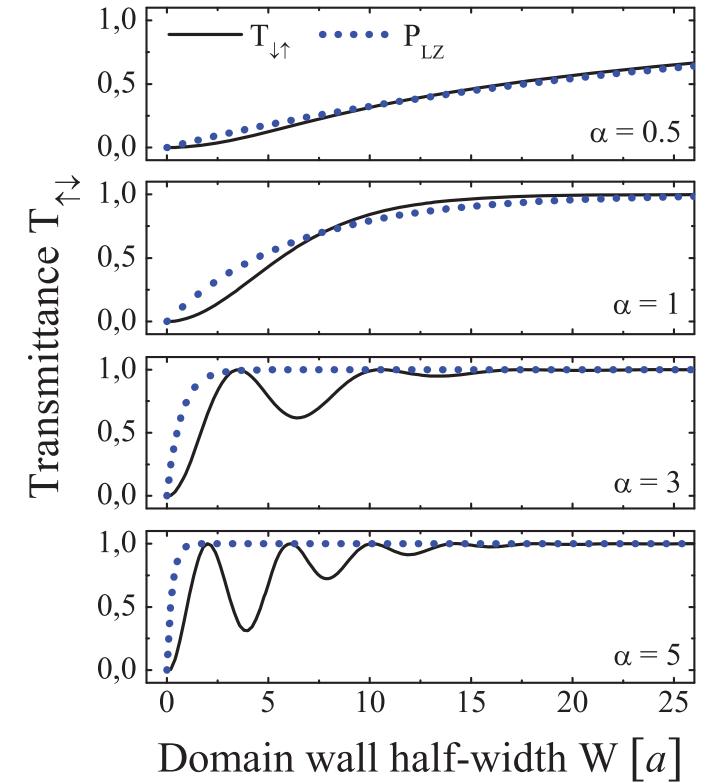

Fig. 3: (Color online) The transmittance $T_{\downarrow \uparrow} v s$. the DW halfwidth $W$ for different $\alpha$. The energy is measured in units of $V$. The Zeeman splitting is $2 \Delta_{0}=0.2 \mathrm{~V}$ and $V_{\uparrow \downarrow}=\alpha \Delta_{0}$. The transition probabilities predicted by the Landau-Zener formula $P_{L Z}$ are shown with dashed lines.

Here, we can use $E(x)=E_{\uparrow}(x)-E_{\downarrow}(x)$ and $x \simeq v_{F} t$ to evaluate the derivative. The adiabaticity parameter $2 \pi\left|V_{\uparrow \downarrow}\right|^{2} /(\hbar \mathrm{d} E / \mathrm{d} t)$ describes a fully adiabatic transition if it is much greater than 1 resulting in $P_{\downarrow \uparrow} \lesssim 1$, while the opposite limit is a diabatic process where $P_{\downarrow \uparrow} \gtrsim 0$.

We will consider a wave packet with $\varepsilon_{F} \gg \Delta_{0}$ and $v_{F}(x) \simeq 2 a V / \hbar$. Hence, the time of transit through the $\mathrm{DW}$ is $\tau_{W}=2 W / v_{F}$. We can relate the DW crossing with the LZ problem identifying

$$
\begin{aligned}
\frac{\mathrm{d} E}{\mathrm{~d} t} & =\frac{\mathrm{d} E}{\mathrm{~d} x} \frac{\mathrm{d} x}{\mathrm{~d} t} \\
& \simeq \frac{2 \Delta_{0}}{W} v_{F},
\end{aligned}
$$

where $E(x)$ is calculated as in the LZ formula, being $E_{\uparrow}$ and $E_{\downarrow}$ obtained from eq. (5). Thus, the adiabaticity parameter results as $\pi(a / W)\left|V_{\uparrow \downarrow}\right|^{2} /\left(\Delta_{0} V\right)$, where $V_{\uparrow \downarrow}=\alpha \Delta_{0}$. We will choose to control the adiabaticity of the crossing by changing $W$.

Numerical results. - We will use eq. (13) to evaluate the spin-dependent transmittance, $T_{\downarrow \uparrow}$, that describes the spin-flip process. In fig. 3 we compare $T_{\downarrow \uparrow}$ with the spinflip probability of the LZ problem. Both are shown as a function of the parameter $W$. We show four different DW characterized by $\alpha=0.5,1,3$ and 5 . In all cases we consider $\Delta_{0}=0.1$ and $V=1$.

The two upper panels of fig. 3 may be associated to the $\alpha \leq 1$ regime. At the center of the DW, $\left.V_{\uparrow \downarrow}(x)\right|_{x=0}=$ $V_{\uparrow \downarrow}^{\max }=0.5 \Delta_{0}$ and $V_{\uparrow \downarrow}^{\max }=1 \Delta_{0}$. If the DW is abrupt, i.e. $W \simeq 0$, the electrons keep their initial spin orientation, thus $T_{\downarrow \uparrow} \simeq 0$. This coincides with the regime where the giant magnetoresistance arises from the scattering at the DW [4]. On the other hand, if the DW width is broad enough, the electrons tend to change their spin orientation $T_{\downarrow \uparrow} \simeq 1$, preventing the magnetoresistance. Considering the overall dependence on $W$ for $\alpha \leq 1$, we appreciate that there is a fair correspondence between the transmittance and the LZ probability that improves as $\alpha$ becomes smaller. Even when there are small discrepancies, these can be attributed to the complexity inherent in our problem, where the "perturbation" $V_{\uparrow \downarrow}$ gradually turns on while the levels become degenerate at the DW center. This exceeds the simplicity of the LZ model.

The two lower panels of fig. 3 correspond to a $\alpha>1$ regime, with $V_{\uparrow \downarrow}^{\max }=3 \Delta_{0}$ and $V_{\uparrow \downarrow}^{\max }=5 \Delta_{0}$. In both cases the transmittances oscillate as a function of $W$. This might suggest an analogy to the Fabry-Pérot interferences in tunneling problems. In the case of ref. [10] this phenomenon is justified because their potentials have slopes with discontinuities at the DW. However, here we consider a high-energy problem with smooth potential barriers. Therefore, it always results that $T_{\downarrow \uparrow}+T_{\uparrow \uparrow} \lesssim 1$ and the reflectances are nearly zero. Hence, the Fabry-Pérot interferences are discarded as possible origin of the observed oscillations. Instead, while the electron moves across the DW its spin oscillates between the states $|\uparrow\rangle$ and $|\downarrow\rangle$ driven by the "perturbation" $V_{\uparrow \downarrow}$. This is consistent with the fact that the spin precesses around the local field. This is called Larmor precession for a semiclassical spin and Rabi oscillation for a spin- $(1 / 2)[19]$. This $\pi(a / W)\left|V_{\uparrow \downarrow}\right|^{2} /\left(\Delta_{0} V\right) \lesssim 1$ regime, contrasts with the adiabatic transition where the electron's spin simply remain aligned with the local magnetic field while it crosses the DW. However, since both DWs are smooth, the oscillation frequency varies continuously and thus it is not obvious that well-defined Rabi oscillations would show up.

Transport in the regime of $\alpha>1$ : interferences as Rabi oscillations. - In the DW, the Zeeman energies in the laboratory frame are degenerate while the coupling $V_{\uparrow \downarrow}(x)$ is maximum $V_{\uparrow \downarrow}=\left.V_{\uparrow \downarrow}(x)\right|_{x=0}=\alpha \Delta_{0}$. Locally, this can be seen as a two-level system undergoing a Rabi oscillation with period

$$
\tau_{R}=\frac{\pi \hbar}{V_{\uparrow \downarrow}} .
$$

Therefore, the length traveled by the electron during that Rabi cycle is

$$
L_{R}=\tau_{R} v_{F}=2 \frac{\pi a V}{\alpha \Delta_{0}}
$$

where $v_{F} \simeq 2 a V / \hbar$. We adopt the term "Rabi oscillation" to emphasize that the spin-( $1 / 2)$ is in an oscillating superposition of its two possible projections.

We analyze the spin-flip transmittances in terms of the length scales estimated above. In the case of the two upper panels of fig. $3, \alpha=0.5$ and $\alpha=1$, the Rabi oscillation might have, according to eq. (17), characteristic lengths $L_{R} \simeq 125.6 a$ and $L_{R} \simeq 62.8 a$, respectively. These 


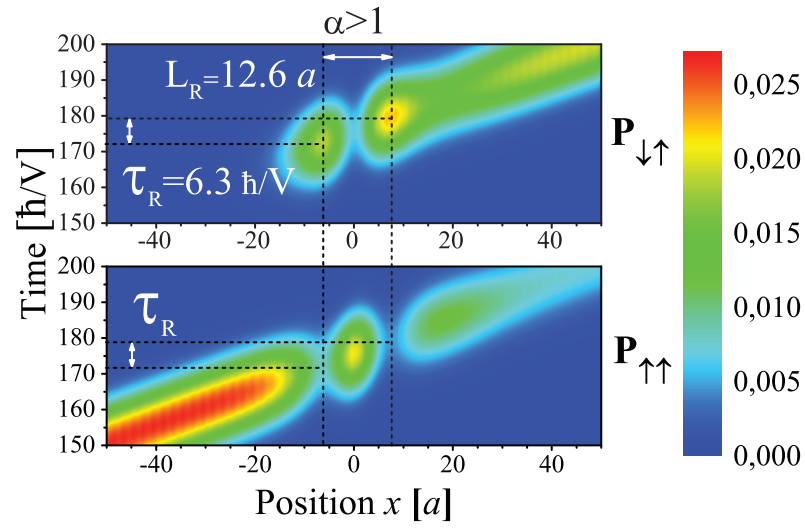

Fig. 4: (Color online) Probability (color scale) for a $\downarrow$ spin orientation (upper panel) or $\uparrow$ spin orientation (lower panel) as a function of time and position, given that the initial electron state on the left has $\uparrow$ spin and moves with the Fermi velocity $v_{F}=2 a V / \hbar$. Here, $\Delta_{0}=0.1 V, W=8 a$ and $\alpha=5$. The midpoint of the DW is located at $x=0 a$. The probability oscillations confirm that Rabi oscillations are present. The vertical and horizontal short-dashed lines come into contact at the maxima and minima of the oscillations. From these, the magnitudes of the period and oscillation characteristic length can be inferred. These coincide with those predicted by the eqs. (16) and (17), $\tau_{R}=6.3 \hbar / V$ and $L_{R}=12.6 a$ as shown by the white arrows.

are much longer than the DW width needed for an adiabatic spin-flip. This explains the absence of oscillations and the applicability of the LZ formula. In contrast, the $\alpha=3$ and $\alpha=5$ cases, shown in the two lower panels, the spin-flip transmittances present oscillations with characteristic lengths $L_{N} \simeq 8 a$ and $L_{N} \simeq 4 a$, respectively. According to our hypothesis of transmittances modulated by Rabi oscillations, the spacing between two consecutive local minima must be $L \simeq 10.5 a$ and $L \simeq 6.3 a$, respectively. The discrepancy between our naïve prediction and the numerical results is justified by the fact that the Rabi length is not a perfectly defined magnitude in our smooth DW model. This is because $V_{\uparrow \downarrow}$, and hence the involved periods, change as the electron moves through the DW.

In order to confirm the Rabi oscillation hypothesis, we analyze the dynamical evolution of the electron's spin implementing a unitary algorithm based on the Trotter approximation [20]. We consider an initial Gaussian wave packet with spin $|\uparrow\rangle$ and a well-defined momentum at the band center. This last condition avoids undesired effects of dispersion. During the electron's transit through the DW, the spin projection tries to follow the magnetic field and thus the final spin projection depends on $W$.

In fig. 4 we show the probability densities $P_{\downarrow \uparrow}(x, t)$ and $P_{\uparrow \uparrow}(x, t)$ for $\alpha=5$. Here, the second subscript is the initial spin, while the first one indicates the corresponding spin projection at time $t$. The upper panel shows the probability for the $|\uparrow\rangle$ spin projection, while the lower panels show the $|\downarrow\rangle$ spin projection. The Zeeman splitting inside

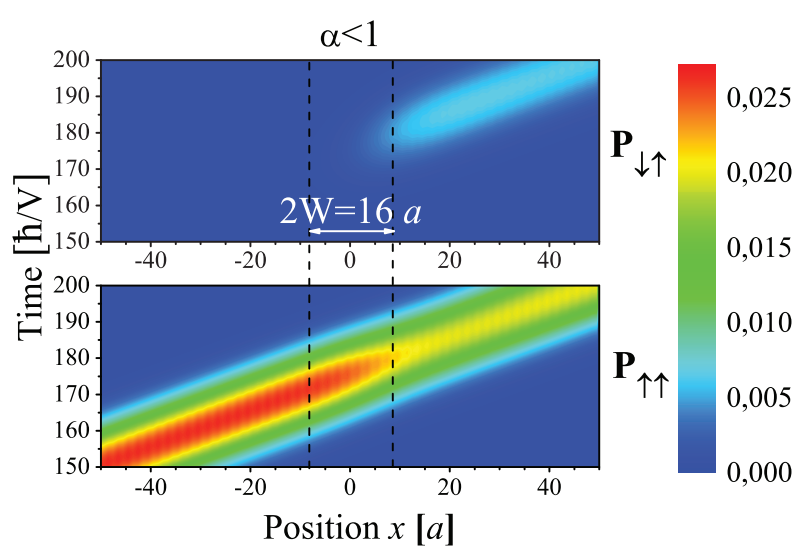

Fig. 5: (Color online) Probability (color scale) for a $\downarrow$ spin orientation (upper panel) and $\uparrow$ spin orientation (lower panel) as a function of time and position, for $\Delta_{0}=0.1 \mathrm{~V}, \mathrm{~W}=8 \mathrm{a}$ and $\alpha=0.5$. The dashed lines indicate the effective width of the DW, $2 W$. The Rabi oscillations are not developed.

the domains is $2 \Delta_{0}$, with $\Delta_{0}=0.1 \mathrm{~V}$. The DW center is placed at $x=0 a$ and its width is $2 W=16 a$, which implies an adiabaticity parameter of about 1 . According to fig. 3 , this $W$ ensures some oscillations in the transmittance. The intensity plot is consistent with the fact that as the electron moves through the DW, the probability of finding the electron with the spin-up projection decreases while the complementary spin-down density increases. Subsequently, an increase in the spin-up probability is produced while a decrease occurs for the opposite spin projection. This cycle is repeated until the electron reaches the end of the DW. This oscillation between the two spin projections is identified with a Rabi oscillation. The observed period and characteristic length are in full agreement with those given by eqs. (16) and (17), $\tau_{R}=6.3 \hbar / \mathrm{V}$ and $L_{R}=12.6 a$, respectively. These magnitudes are drawn in fig. 4.

The same analysis is performed for a $\alpha=0.5 \mathrm{DW}$ and shown in fig. 5 . This $\alpha$, together with $2 W=16 a$, implies a low adiabaticity parameter of about 0.01. Again, the final electronic state $\left|\varphi_{R}\right\rangle$ in the right domain, after traversing the DW, is a superposition of the two spin projections. The probability of finding the state $|\downarrow\rangle$, is consistent with the transmittance shown in fig. 3 and the LZ prediction: $T_{\downarrow \uparrow} \equiv\left|\left\langle\downarrow \mid \varphi_{R}\right\rangle\right|^{2}=P_{\downarrow \uparrow}$ and $T_{\uparrow \uparrow}=\left|\left\langle\uparrow \mid \phi_{R}\right\rangle\right|^{2}=P_{\uparrow \uparrow} \simeq$ $1-T_{\downarrow \uparrow}$. As consequence of the DW smoothness there are no significative reflections. In contrast to the previous case, we see that the time oscillations are not developed.

While in the present work we just analyzed a single incoming wave vector, considering a metallic wire would involve integrating, up to the Fermi energy, over transversal channels equivalent to those as described here. This could smear out the Rabi oscillations reported here and one would need a proper design to overcome this difficulty. However, in magnetic semiconducting waveguides, the relevant role of lateral quantization leaves the considered model as a realistic description [8]. 
Conclusion. - In this letter we explored the quantum phenomena associated to spin-dependent transport in the presence of a smooth magnetic inhomogeneity, much as a DW in a magnetic nanowire. For this purpose, we extended the Cabrera-Falicov model to account for modulations on the magnetic-field intensity at the DW. The physics we described is not restricted to this case. Indeed, our results and strategies remain valid for other situations, such as magnetically modulated semiconducting structures [7]. There, spectral modulations are described by variants of eq. (4). In ref. [8] a situation assimilable to our $\alpha<1$ is presented for a spin transistor based on helical magnetic fields.

We showed that, for $\alpha \leq 1$, the spin-dependent transport across the magnetically modulated region is fairly described by the LZ formula. LZ applies to the whole dynamical range, from diabatic to fully adiabatic crossing. We showed that, by performing the appropriate mapping of the relevant variables, LZ yields a quite fair description under well-defined conditions for the perturbation. For $\alpha>1$, we found that conductance has quantum interferences which manifest as oscillations as a function of the DW width. These cannot be assigned to Fabry-Pérot interferences. By performing a dynamical study of the tunneling process, we showed that a spin-polarized wave packet propagating across the DW, can be seen as a twolevel system undergoing Rabi oscillations.

A possible experimental set up to test the discussed effects in all regimes could be a linear semiconducting waveguide in the presence of a locally modulated field. In such a case, few conducting channels are enabled by a gate voltage that also controls the carrier's wavelength. Finally, the dynamical description of the transport problem as presented here, may prove useful for the converse problem: i.e. evaluating the dynamics of a DW under pulsed electrical currents. This may extend the interest of our strategy to study a problem of growing interest: electrically driven domain-wall-based memories in quasione-dimensional (1D) magnetic wires [21].

In summary, for the perturbative regime $(\alpha<1)$, we probed a definite connection between steady-state spindependent transport across a magnetically modulated region and the time-dependent Landau-Zener problem. In the strong perturbation regime $(\alpha>1)$, we showed that the steady-state conductance presents interferences. We probed that they arise from Rabi oscillations, by performing a time-dependent calculation.
$* * *$

This work was performed with the financial support from CONICET, ANPCyT, SeCyT-UNC and MinCyTCor.

\section{REFERENCES}

[1] Žutić I., Fabian J. and Das Sarma S., Rev. Mod. Phys., 76 (2004) 323.

[2] Schliemann J., Egues J. C. and Loss D., Phys. Rev. Lett., 90 (2003) 146801.

[3] Reynoso A. A., Usaj G., Balseiro C. A., FeinBerg D. and Avignon M., Phys. Rev. Lett., 101 (2008) 107001.

[4] Fert A., Rev. Mod. Phys., 80 (2008) 1517.

[5] Steil S., Großmann N., Laux M., Ruffing A., Steil D., Wiesenmayer M., Mathias S., Monti O., Cinchetti M. and Aeschlimann M., Nat. Phys., 9 (2013) 242.

[6] Dediu V. A., Nat. Phys., 9 (2013) 210.

[7] Betthausen C., Dollinger T., Saarikoski H., Kolkovsky V., Karczewski G., Wojtowicz T., Richter K. and Weiss D., Science, 337 (2012) 324.

[8] Satrikoski H., Dollinger T. and Richter K., Phys. Rev. B, 86 (2012) 165407.

[9] Imry Y. and Landauer R., Rev. Mod. Phys., 71 (1999) S306.

[10] Gopar V. A., Weinmann D., Jalabert R. A. and Stamps R. L., Phys. Rev. B, 69 (2004) 014426.

[11] Falloon P. E., Jalabert R. A., Weinmann D. and Stamps R. L., Phys. Rev. B, 70 (2004) 174424.

[12] Cabrera G. G. and Falicov L. M., Phys. Status Solidi B, 61 (1974) 539.

[13] Czerner M., Yavorsky B. Yu. and Mertig I., Phys. Rev. B, 77 (2008) 104411; Burton J. D., Sabirianov R. F., Jaswal S. S. and Tsymbal E. Y., Mryasov O. N., Phys. Rev. Lett., 97 (2006) 077204.

[14] Büttiker M., Phys. Rev. Lett., 57 (1986) 1761.

[15] Pastawski H. M. and Medina E., Rev. Mex. Fis., 47 (2001) 1.

[16] Levstein P. R., Pastawski H. M. and D'Amato J. L., J. Phys.: Condens. Matter, 2 (1990) 1781.

[17] Pastawski H. M., Physica B, 398 (2007) 278.

[18] Zener C., Proc. R. Soc. Lond. A, 137 (1932) 696.

[19] Cohen-Tannoudji C., Grynberg G. and Dupont-Roc J., Atom-Photon Interactions: Basic Processes and Applications (Wiley, New York) 1992, p. 10.

[20] De Raedt H., Annu. Rev. Comput. Phys., 4 (1996) 107.

[21] Thomas L., Hayashi M., Jiang X., Moriya R., Rettner C. and Parkin S., Science, 315 (2007) 1553. 